\title{
El uso de material didáctico y las tecnologías de información y comunicación (TIC's) para mejorar el alcance académico
}

María Guadalupe Bautista Sánchez¹, Aldo Raudel Martínez Moreno

y Reynaldo Hiracheta Torres ${ }^{1}$

\section{Resumen}

Sin lugar a dudas, una de las tareas más complejas y que más tiempo consume enInternet está revolucionando al mundo pero eso no es un secreto. El rápido progreso de las Tecnologías de la Información y Comunicación (TIC's) continúa modificando la forma de elaborar, adquirir y transmitir los conocimientos, es por eso que los sistemas educativos con sus modelos y estrategias se han visto en la necesidad de adaptarse a una sociedad que está cada vez más sumergida en las TIC's, ya que éstas han brindado posibilidades de renovar el contenido de los cursos y métodos pedagógicos.

La educación se vuelve cada vez más competitiva y para alcanzar un mejor nivel educativo se requiere del apoyo de recursos que nos ayuden en el proceso de enseñanza de los estudiantes, como lo son los materiales didácticos, su uso tiende a guiar y motivar al estudiante en la construcción del conocimiento, es decir, que sirvan de apoyo en el proceso de aprendizaje de los estudiantes mediante publicaciones de sistemas pedagógicos innovadores utilizando herramientas tecnológicas.

Palabras clave: TIC's, Materiales Didácticos, Uso.

1 Facultad de Ingeniería Mecánica y Eléctrica de la Universidad Autónoma de Nuevo León (UANL), México. 


\section{Abstract}

Internet is revolutionizing the world but that's no secret. The rapid progress of Information Technology and Communication (TIC's) continues to modify how to develop, acquire and transmit knowledge, that is why education systems with their models and strategies have been the need to adapt to a society that is increasingly immersed in TIC's, as they have given you opportunities to innovate on course content and teaching methods.

Education is becoming more competitive and to achieve a better educational level requires the support of resources to help us in the process of teaching students , such as teaching materials, their use tends to guide and motivate the student in the construction of knowledge, ie, that support the learning process of students through innovative teaching publications systems using technological tools.

Key words: TIC's, Educational Materials, Use. 


\section{Introducción}

Todos queremos vivir en un mundo mejor, tener todas las comodidades que la sociedad presenta hoy en día, tener todo a nuestro alcance y realizar el más mínimo esfuerzo en cada una de nuestras tareas diarias haciéndonos cada día más dependientes del uso de los diferentes medios electrónicos y el Internet, ya solo basta tener a la mano un celular para efectuar compras desde nuestra casa, revisar nuestros estados de cuenta y realizar pagos o transferencias bancarias desde el lugar más cómodo de nuestra habitación, incluso para saber dónde nos encontramos por medio del uso del GPS, y algunas otras cosas más que nos resulta común hacer en nuestra vida cotidiana como comunicarnos con nuestras familias y amigos a través de las distintas redes sociales.

Por lo que a cada momento hacemos uso de estos recursos, ya no hay duda de que todas estas herramientas se han vuelto cada vez más indispensables, sobre todo para las diferentes organizaciones educativas de todo el mundo, que en la actualidad se enfrentan al desafío de utilizar las Tecnologías de la Información y Comunicación para proveer a sus alumnos con las herramientas y conocimientos necesarios que requieren en el siglo XXI.

Uno de los problemas que se han enfrentado las instituciones educativas no ha sido la integración de las TIC's a los procesos de enseñanza-aprendizaje, porque una vez que se posee la tecnología, el tema que surge es cómo los profesores pueden hacer uso de ella, y de qué manera la van a integrar a sus métodos de enseñanza, ya que su formación TIC's es deficiente.

Para muchos docentes el uso de la TIC's implica ciertas desventajas, tales como aprender a usar las tecnologías, actualizar los equipos y programas, sobre todo, implica ocupar un tiempo fuera del lugar de trabajo, el cual muchos docentes no pretenden acceder. Según Mumtag (2005), los principales factores que influyen en el uso de las TIC's por parte de los docentes son: el acceso a este tipo de recursos, calidad de software y equipos, facilidad o simplicidad de uso, incentivo para cambiar las prácticas pedagógicas usando tecnología, el apoyo y solidaridad de las escuelas para usar las TIC's en el currículo, las políticas nacionales y locales sobre TIC's, compromiso con la superación profesional, y la capacitación formal recibida en el uso de las TIC's. (Gallardo \& Buleje, 2010)

Las TIC's en la mayoría de los casos no han sido utilizadas como una herramienta esencial para tener acceso a la información. Es preciso considerar que las nuevas formas de concebir la enseñanza y el aprendizaje con el apoyo de los materiales didácticos, están provocando diversas actitudes y opinión frente al uso y aprovechamiento para lograr un rendimiento académico óptimo. Por tanto, los profesores deben poseer los niveles de conocimiento y habilidades necesarias para acompañar a sus estudiantes durante este proceso, y asumir que la incorporación de 
estas herramientas tecnológicas facilitara su quehacer pedagógico y administrativo, además de enriquecer los ambientes de aprendizaje.

Los alumnos necesitan utilizar los diversos medios tecnológicos para formar su futuro profesional. Esta situación provoca impulsar nuevas y variadas maneras de ejercer la docencia, saber aprovechar las nuevas tecnologías de información y comunicación, que la mayoría de los estudiantes ya mantiene un dominio sobre ellas.

\section{Como han influido las tic's en la enseñanza y en el aprendizaje}

Las Tecnologías de Información y Comunicación (TIC's) son una especie de unión entre tres tecnologías que han sido inventadas en el siglo XX, la Informática, las Telecomunicaciones y los Medios Audiovisuales.

Las TIC's son herramientas que nos permiten acceder a gran cantidad ilimitada de información, por ejemplo pensemos en un libro y en un video, antes se tenía información limitada en cuanto a las páginas de los libros, y en el video a la longitud del mismo. Pensemos también en una biblioteca, en una biblioteca por mucha cantidad de libros que haya, la cantidad de información disponible a la cual podemos acceder es limitada. Sin embargo hoy en día con el Internet, la cantidad de información a la que se puede acceder y almacenar es infinita.

Este tipo de tecnologías permiten que haya interacción entre el humano y las máquinas, es decir que sean reactivas a las acciones de los humanos, el videojuego por ejemplo es una de las tecnologías más características de este tipo de interacción, es algo que no permite un libro, que no permite la televisión, somos en estos últimos casos receptores de información y poco podemos hacer con este tipo de tecnologías. Sin embargo, con las TiC's podemos elegir en función de ciertas características que la máquina, según lo que hagamos reaccione de modo distinto y esto para las personas resulta muy atractivo.

Las TIC's son además de un recurso para obtener información, un recurso potente, valioso para que las personas nos podamos comunicar con nosotros mismos a través de aparatos móviles, correo electrónico, los foros de debate, los chats, entre otras cosas más.

En el mundo educativo podemos encontrar infinidad de aplicaciones de las TIC's, desde la creación de portales o webs educativas, la creación de aulas virtuales de enseñanza-aprendizaje, la videoconferencia, software para la educación y lógicamente todo el conjunto de material didáctico que tiene un soporte de disco o de multimedia educativo que actualmente se distribuye a través de Internet.

En las últimas décadas se han ido incorporando a la sociedad nuevos y mejores recursos tecnológicos que ponen de manifiesto la manera de llevar a cabo los procesos y modelos de enseñanza y aprendizaje. Con la aparición de las computadoras portátiles 
y el Internet, se hizo posible el intercambio y el fácil acceso a fuentes de información, trayendo consigo importantes cambios en el ámbito educativo.

Por ello la educación debe replantear sus objetivos, sus metas, sus pedagogías y sus didácticas si quiere cumplir con su misión en el siglo XXI, brindar satisfactores a las necesidades del hombre, como dice Bill Gates en lo que trae el futuro "Las mismas fuerzas tecnológicas que harán tan necesario el aprendizaje, lo harán agradable y práctico. Las corporaciones se están reinventando en torno a las oportunidades abiertas por la tecnología de la información, las escuelas también tendrán que hacerlo". (Ossa, 2002)

En la Universidad, al igual que en otros ámbitos, el uso constante de las tecnologías ha estado dictado por su evolución y desarrollo, $\mathrm{y}$, aunque se han aplicado a la educación desde mucho tiempo atrás, es a partir de la década de los ochenta cuando comienza su apogeo.

La forma en la cual las tecnologías se abrieron paso muy rápidamente en este ámbito de la educación, ha provocado cambios en las formas de enseñar a los alumnos, en la actualidad es evidente que existe un problema en el aprendizaje de los estudiantes, esto puede deberse a diversos factores, tales como el nivel intelectual o desinterés hacia la escuela por parte de los alumnos, esta situación está ligada también a la forma de enseñar del profesor y a sus estrategias de enseñanza. Otro factor importante que impide tener un buen aprendizaje es la distracción que existe en el uso constante de las nuevas tecnologías por parte de los alumnos (Celulares, Laptops, Tablet, etc.). Ya que ellos muestran una gran destreza a la hora de utilizar este tipo de tecnología se deberían emplear nuevas estrategias de enseñanza donde el estudiante y el docente empleen su uso para generar distintos materiales didácticos que faciliten la obtención de conocimientos, habilidades y aptitudes para que puedan alcanzar un mejor nivel educativo.

Deben crearse métodos para que el estudiante aprenda y no para que el profesor enseñe; para esto, la tecnología propicia el medio, el resultado son los entornos de aprendizaje virtuales como una nueva forma organizativa de la enseñanza que permite al profesor, de una manera pedagógica, gestionar y diseñar contenidos para ampliar sus conocimientos sobre algún tema, pero que, además, posibilita diseñar actividades y evaluaciones que propicien una retroalimentación y comprobación de los objetivos propuestos, y todo sobre la base de las TIC's, sea mediante los foros, el correo electrónico, los weblogs, los wikis o los ejercicios interactivos, que flexibilizan el proceso de enseñanza aprendizaje para el estudiante y permiten que este se sienta como el actor central del proceso. (Rodríguez1, 2007)

Como dice Einstein: "Es una locura seguir haciendo lo mismo y esperar resultados diferentes".

El uso de las diversas herramientas TIC's en el entorno educativo otorga no sólo múltiples ventajas sino que ofrece una alta flexibilidad de tiempo y espacio, 
permiten crear materiales didácticos que apoyen el aprendizaje de los estudiantes así como también mejorar la calidad de la educación y amplían las oportunidades de acceso al conocimiento.

\section{Material didáctico para la educación}

Las situaciones organizativas además de las personas, el profesor, los estudiantes, además de los contenidos que se enseñan, de las actividades, de la metodología que se desarrolla, están presentes un conjunto de medios, de materiales que permiten trabajar, aprender, y diseñar. Esos son los materiales didácticos.

Los materiales didácticos son un tipo particular de los medios de información, de los medios de comunicación que existen en nuestra sociedad. Un medio vendría siendo una especie de soporte físico que registra, que guarda información que no está almacenada en la mente humana, y esto es consustancial con el propio desarrollo del sujeto humano como ser civilizado, los primeros vestigios que hemos encontrado en la historia, han sido el intentar dejar registro del pensamiento, de las ideas, de la imaginación humana a través de huellas en las piedras, el arte rupestre es el mejor ejemplo de ello.

Hace más de 2,000 o 3,000 años antes de Cristo fuimos capaces de registrar el pensamiento humano a través de códigos, de símbolos, colecciones de signos que hoy conocemos como la escritura, y la escritura es algo complejo, algo abstracto que exige la preparación y dominio tanto del sujeto que escribe como del sujeto que tiene que decodificar la información, ya que éste último tiene que dominar los símbolos del alfabeto para poder entender el significado.

Definir el concepto de material didáctico es una tarea que resulta difícil, ya que existen varias definiciones al respecto, según Cabrero (2001) los medios o materiales didácticos son elementos curriculares que por sus sistemas simbólicos y estrategias de utilización, propician el desarrollo de habilidades cognitivas en los sujetos, en un contexto, determinado, facilitando y estimulando la intervención mediada sobre la realidad, la captación y comprensión de la información por el alumno y la creación de entornos diferenciados que propician aprendizajes.

Podemos decir entonces que un material didáctico es el conjunto de medios materiales que intervienen y facilitan el proceso de enseñanza-aprendizaje, estos materiales pueden ser tanto físicos como virtuales, que despiertan el interés y capten la atención de los estudiantes, que presenten información adecuada con experiencias simuladas cercanas a la realidad, que vivifican la enseñanza influyendo favorablemente en la motivación, retención y comprensión por parte del estudiante, facilitando la labor docente por ser sencillos, consistentes y adecuados a los contenidos. 
Los primeros materiales didácticos que existieron se trataban de ayudas visuales. Los antiguos griegos descubrieron que la tierra era esférica en el año 150 a.C. utilizando un globo terráqueo, lo que significa que el globo terráqueo se encontraba entre los primeros medios de enseñanza. Otro de los primeros materiales didácticos que existieron fue el abecedario, el cual desde mediados del siglo XV se ocupaba en las escuelas inglesas hasta principios del siglo XIX, en la época colonial de los Estados Unidos. El pizarrón, una herramienta esencial de enseñanza, también se encuentra entre los primeros materiales didácticos, el pizarrón produjo cambios en la educación en 1801 facilitando el trabajo a los maestros.

Pero que hace que los materiales didácticos sean tan importantes, pues bien, la importancia radica en que son los medios o recursos que sirven para aplicar una técnica concreta en el ámbito de un método de aprendizaje determinado, entendiéndose por método de aprendizaje el modo, camino o conjuntos de reglas que se utilizan para obtener un cambio en el comportamiento de quien aprende, y de esta forma que potencie o mejore su nivel de competencia a fin de desempeñar una función productiva. (MUÑOZ, 2012)

\section{Como elegir el materiale didactico que sirva de apoyo en el proceso enseñanza-aprendizaje}

En la actualidad, adaptarse a los planes de estudios y elaborar un excelente material didáctico para cumplir con los objetivos del proceso de enseñanzaaprendizaje planteados en clase no es tarea fácil como puede parecer.

Los materiales visuales con frecuencia transmiten ideas y contenidos más fácilmente que las descripciones verbales, y proporcionan mejoras importantes de aprendizaje en el aula. Un curso enriquecido con gráficos, diagramas, fotografías, presentaciones visuales, videos y mapas se absorbe más fácilmente por los estudiantes. Aunque los materiales visuales no son un sustituto para una conferencia convincente y atractiva, pueden ayudar a los estudiantes con los contenidos y liberarlos de tomar notas, que en algunos casos puede provocar que se pierda puntos importantes.

Los estudiantes pueden usar la tecnología ya que puede mejorar bastante el aprendizaje de un estudiante al ayudarle a procesar información de una manera integral, al contrario de simplemente ver datos y figuras en una prueba. Las herramientas tecnológicas como e video, multimedia, internet y equipo en general han servido de apoyo en el proceso de enseñanza-aprendizaje y han sido elaboradas por el profesor, ayudando a mejorar la calidad de los procesos de enseñanzaaprendizaje mediante la aplicación de sistemas pedagógicos innovadores.

Los profesores deben tener materiales didácticos innovadores en donde se 
incorporen recursos tecnológicos, pues estos traen consigo nuevas oportunidades para el entorno educativo y despiertan el interés de los estudiantes y al mismo tiempo hacen que los estudiantes se encuentren motivados por su propio aprendizaje con las actividades que se les propongan, ya que los estudiantes aprenden con mayor entusiasmo cuando se involucran y les es más significativo ya que encuentran trabajando con herramientas que conocen y utilizan diariamente, el docente debe ser original en el entendido de anular lo tradicional, ser creativo, auténtico e innovador.

\section{En que benefician los materiales didacticos en el proceso enseñanza-aprendizaje}

Son diversas las funciones que los materiales didácticos pueden tener, una de las principales es el reforzar lo que se ha enseñado en clase, de modo que facilite el aprendizaje de los alumnos.

Los materiales didácticos, la mayoría, proporcionan información, a través de libros, videos y programas informáticos, guiando al estudiante y ayudándolo a crear y aplicar nuevos conocimientos ejercitando habilidades.

No obstante, se debe tener en cuenta que los materiales didácticos no solamente sirven para transmitir información sino también funcionan como mediadores entre la realidad y los estudiantes, y mediante sus sistemas simbólicos desarrollan habilidades cognitivas en sus usuarios.

Algunas otras funciones de los materiales didácticos son Motivar, Facilitar la adquisición de nuevos conocimientos y Apoyar la evaluación y el Reforzamiento del Aprendizaje.

\section{Motivacion}

En su libro "Como abrir mentes cerradas", William (1997) menciona, que el Deseo es la más poderosa fuerza motivadora de la vida humana. Las acciones humanas brotan de los deseos humanos. De hecho solo existen dos razones por las cuales una persona cualquiera se siente impulsada a hacer algo:

1. Para obtener algo que desea y que no tiene.

2. Para evitar perder algo que posee y que desea conservar.

Con lo expresado anteriormente, me refiero más que nada a la Motivación, esa chispa que nos lleva a poder cumplir lo que tanto deseamos, de ello se desprende nuestra capacidad de comprensión que tengamos para alcanzar los deseos humanos, es decir, los materiales didácticos contribuyen a generar en los estudiantes expectativas sobre lo que van aprender, que los impulse a trabajar por el logro de los objetivos. 
Con la Motivación, los materiales didácticos pueden también desarrollar las siguientes funciones:

a) Desarrollan los temas en forma atractiva, interesante y comprensible.

b) Facilitan, mediante procedimientos didácticos, que los estudiantes progresen exitosamente y puedan así conservar y acrecentar las expectativas iniciales.

\section{Facilitar la adquisición de nuevos conocimientos}

Los materiales didácticos participan en la representación de informaciones, posibilitan diversas actividades y experiencias, inducen a la exteriorización de lo aprendido en conductas observables; apoyan los procesos internos de atención, percepción, memorización, transferencia de aprendizaje y otros, como por ejemplo:

a) Presentan la nueva información a través de estímulos variados que atraigan y mantengan la atención de los estudiantes.

b) Durante el desarrollo, presentan los contenidos o informaciones adecuadamente organizados y dosificados; emplean lenguajes comprensibles para los alumnos; proporcionan diversidad de ejemplos, casos, situaciones, modelos de desempeño, etc.

c) Orientan la labor de análisis y síntesis de la información a través de cuadros gráficos, resúmenes, ejemplos y otros.

d) Dan posibilidad para la conducta activa de los estudiantes y la aplicación de lo aprendido mediante ejercicios, problemas, guías de observaciones y de análisis, sugerencias de actividades y otros procedimientos.

\section{Apoyar la evaluación y el reforzamiento del aprendizaje}

Evaluar significa estimar en qué medida el elemento evaluado tiene unas características que se consideran deseables en función de unos criterios que han sido especificados con respecto a un conjunto de normas.

El apoyo a la evaluación se puede hacer mediante prácticas o ejercicios corregidos, pruebas de autoevaluación y otros procedimientos similares, esto permite que cada estudiante compruebe progresivamente sus aciertos y errores.

La corrección de los errores de los estudiantes a veces se analiza de manera explícita (como en el caso de los materiales multimedia que monitorean las acciones de los usuarios) y en otros casos de manera implícita ya que es el propio estudiante que se da cuenta de sus errores.

Con los aciertos, los estudiantes obtendrán el necesario reforzamiento positivo que consolide su aprendizaje e impulse a continuar estudiando.

En sí, corresponde al profesor seleccionar o preparar materiales didácticos que puedan desempeñar determinadas funciones en la enseñanza. 


\section{Importancia del uso de material didáctico en la educación}

Hemos hablado del concepto de los materiales didácticos, de las funciones que éstos desempeñan, sin embargo en donde radica su importancia de utilizarlos si queremos tener un mejor alcance académico.

Con la incorporación de las nuevas tecnologías en todos los aspectos de la vida y la sociedad misma está demandando nuevos modelos de enseñanza y aprendizaje. El modelo de educación centrado en la enseñanza, donde el protagonista es el profesor, deja paso a un sistema basado en el aprendizaje, donde el alumno es el responsable de su propio proceso de aprendizaje y el profesor debe buscar y utilizar la metodología y los medios más adecuados que ayuden al alumno en ese proceso. (García \& Lacleta, 2007).

La utilización de distintos medios de enseñanza permite a los estudiantes aprender en muchos niveles diferentes. Las herramientas tecnológicas pueden emplearse en el sistema educativo como objeto de aprendizaje, como medio para aprender o bien como apoyo al aprendizaje. El término materiales didácticos es un término que se utiliza para referirse a los recursos que los profesores emplean para apoyar su clase.

Los materiales didácticos apoyan el aprendizaje de los estudiantes y el aumento de su éxito, por eso su importancia, porque pueden aumentar el logro estudiantil. Por ejemplo un tema preparado en una diapositiva puede proporcionar al estudiante importantes oportunidades de practicar algo adquirido dentro de la clase, lo que le permite al estudiante explorar de forma independiente. Lo ideal sería que los materiales didácticos utilizados se ajusten al contenido de la clase del profesor.

Resulta evidente considerar siempre el aprendizaje de los estudiantes como centro de interés más relevante dentro del proceso educativo, es por eso que deben utilizarse materiales didácticos que resulten atractivos para los estudiantes.

Internet tiene una amplia gama de recursos para profesores, y la mayoría son gratis, que pueden aumentar significativamente el proceso de enseñanza-aprendizaje alcanzando un mejor alcance académico.

\section{Impacto que tiene el materiale didáctico con el uso de las tic's en la educación}

Las aplicaciones de la tecnología en los niveles educativos han ido evolucionando y se han manifestado rápidamente; en la búsqueda por alcanzar una educación de primer mundo, es factible otorgar mayor énfasis en el recurso humano, creando y proporcionando un enfoque e interacción hacia las nuevas tecnologías.

En muchas instituciones, algunos profesores prefieren continuar con la forma de enseñanza antigua, por lo que aún no están haciendo uso de las nuevas herramientas tecnológicas en el aula de clases y otros lo hacen de manera incorrecta, esto 
demuestra un grave problema al quedar estancados y obsoletos en los cambios tecnológicos, creando con esto un conflicto entre profesor-estudiante por no contar con los medios tecnológicos competitivos.

El docente debe crear y buscar continuamente nuevas ideas y estrategias de intervención e instrumentos de enseñanza que ayuden a los alumnos a sacar el máximo partido a sus posibilidades. Cabe mencionar que la incorporación del uso de las Herramientas de Tecnologías de la Información y Comunicación en las aulas permite nuevas formas de acceder, generar y transmitir información y conocimientos, teniendo como beneficio el poder flexibilizar no solo el tiempo, sino el espacio en el que se desarrolla la acción educativa.

Igualmente debe tenerse en cuenta que las posibilidades que nos brindan las nuevas tecnologías como herramienta didáctica, son de igual importancia y es necesario aprovechar todas sus potencialidades para formar seres humanos más justos, más capaces, más cooperativos, los que nos llevaría a afirmar que lo importante no es la tecnología como tal sino que los actores formadores puedan hacer del elemento tecnológico, para humanizarla. (Ossa, 2002)

Las instituciones que enseñen a los estudiantes a hacer un buen uso significativo de las herramientas TIC's y aprovechar los sistemas de apoyo a la cognición, es decir todos esos materiales didácticos, les abrirán las puertas a nuevas posibilidades de acceso a un mayor flujo de información, y mayores oportunidades laborales.

Las TIC's aportan también un espacio que puede integrarse y complementar las tareas del aula, especialmente en la experimentación y aprendizaje sobre modos de expresarse, de comunicarse y de hacerse visible.

\section{Conclusiones}

No hay duda de que el uso de material didáctico facilita la enseñanza y constituye un elemento auxiliar en el proceso de aprendizaje funcionando como mediador en la educación de los estudiantes.

Es por eso que deben utilizarse materiales didácticos que se presenten mediante las herramientas tecnológicas que son de uso común para los estudiantes, materiales innovadores bien pensados y fabricados especialmente para enseñar y aprender de manera que capten la atención de los estudiantes y les permitan llegar a adquirir determinados conceptos y contribuir así al desarrollo de su pensamiento lógico al mismo tiempo que se sientan motivados con su aprendizaje.

Con el uso de los distintos materiales didácticos a través de las TIC's se puede brindar una educación de calidad a un mayor número de estudiante, la institución educativa debe estar preparada para ofrecer esta educación, con un equipo físico, técnico y tecnológico, capaz de afrontar los retos de la nueva era. 


\section{Referencias}

Cabero, J. (2001). Tecnología Educativa, Diseño y Utilización de medios en la enseñanza. España: Paidos Iberica, S.A.

Gallardo, L. M., \& Buleje, J. C. (2010). Importancia de las TIC's en la Educación Básica Regular. Investigación Educativa vol. 14, 209-224.

García, P. L., \& Lacleta, M. L.-E. (2007). MOODLE: difusión y funcionalidades. Obtenidodehttp://cvonline.uaeh.edu.mx/Cursos/Maestria/MTE/Gen02/Admon_ aprendizaje/Unidad\%204/Lect_7_Moodle-Difusion_y_funcionalidades.pdf

MUÑOZ, P. A. (2012). Elaboración de material didáctico. Obtenido de http:// www.aliatuniversidades.com.mx/bibliotecasdigitales/pdf/Derecho_y_ ciencias_sociales/Elaboracion_material_didactico.pdf

Ossa, G. C. (mayo de 2002). Tendencias Educativas para el siglo XXI; Educación virtual, onliney@learning; Elementos para la discusión. Obtenido de http:// edutec.rediris.es/Revelec2/revelec15/cardona.pdf

Reilly, W. J. (1997). Cómo abrir mentes cerradas. México, D.F.: Litografía Joman, S.A. DE C.V. Comonfort No. 48.

Rodríguez1, I. M. (agosto de 2007). Herramientas para la producción de materiales didácticos para las modalidades de enseñanza semipresencial y a distancia. Obtenido de http://scielo.sld.cu/scielo.php?script=sci_arttext\&pid $=$ S1024-94352007000800008 